\title{
Das positiv-blickende Selbst als Schauplatz der Armutsbearbeitung
}

\section{Armut im Familienzentrum}

Der Auftrag pädagogischer Institutionen zur Bearbeitung von Armut ist eng verwoben mit normativen Vorstellungen über die bearbeitungsbedürftigen Missstände, "gute" Elternschaft und Kindheit. Im Familienzentrum machen sich die pädagogischen Fachkräfte zur Bearbeitung eines strukturellen Problems wie Armut in erster Linie selbst zum Gegenstand und inszenieren dies verbal. Dadurch werden nicht nur bestimmte wirkmächtige Normen über Kinder (re-)produziert, sondern auch soziale Ungleichheiten.

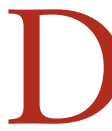
ie Soziale Arbeit ist ebenso wie die Kindheitspädagogik mit normativen gesellschaftlichen Diskursen verknüpft (Otto, Scherr und Ziegler 2010). Peter Cloos et al. (2019, S. 289) bezeichnen die Kindheitspädagogik gar als „rezeptives Feld, in welchem herangetragene Normen und Imperative bereitwillig aufgenommen“ werden.

\section{Kinderarmut - normative Diskurse und rezeptive Felder}

Insbesondere unter dem Auftrag der Bearbeitung von Armut und Devianz durch pädagogische Institutionen werden normative Aspekte formuliert (Otto et al. 2010, S. 142). Kindheitspädagogische Organisationen werden so in unterschiedlicher Weise adressiert, beispielsweise hinsichtlich einer Sozialpädagogisierung durch den Ausbau zu Eltern-Kind-Zentren und der Bereitstellung von

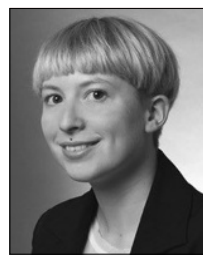

Anja Kerle

Universität Hildesheim, Hildesheim, Deutschland

*1990; Studium Sozialwirtschaft und Soziale Arbeit (M.A), laufende Promotion zu Armut und Familienzentren.

anja.kerle@ites-werkstatt.de

Zusammenfassung Die Fachkräfte und ihre Haltung sind im Feld der Dreh- und Angelpunkt der Armutsbearbeitung. In verschiedenen Sprechweisen inszenieren sie den positiven Blick auf Kinder und Eltern. Armut wird sich dadurch rhetorisch entledigt, während strukturelle Benachteiligungen unerkannt bleiben.

Schlüsselwörter Kinderarmut, Familienzentrum, Performanz, Kindheitspädagogik, Soziale Ungleichheit
Dienstleistungsangeboten für hilfsbedürftige Familien mit dem Ziel einer „Effektivierung der Kontrolle der Risikofamilien “ (Hübenthal 2018, S. 102). Unter der Betonung ihres Bildungsauftrags werden Kindertagesstätten aufgefordert, eine frühe, an wirtschaftlichen Kompetenzprofilen orientierte Bildung für alle Kinder anzubieten, um damit (vermeintlich) Chancengleichheit zu befördern (Hübenthal 2018, S. 117f.). Auch im Sprechen der Fachkräfte in Kindertagesstätten gibt es verschiedene Thematisierungen von Armut (Simon et al. 2019), die jeweils mit Deutungen „guter“ Elternschaft und „würdigen“ und „unwürdigen“ Armen verwoben sind (Simon et al. 2019; Kerle et al. 2019). Offen bleibt bislang allerdings die Frage, wie sich beim Sprechen über Armut Subjektpositionen und Normen verschränken.

Nach einem kursorischen theoretischen Einblick zur Performanz von Normen wird dies anhand von Ergebnissen aus meiner Dissertationsstudie zum Thema Armut in Familienzentren diskutiert.

\section{Die Norm (ver-)sprechen}

Normen besitzen eine orientierende und handlungsanleitende Funktion und entfalten eine produktive und zugleich repressive Macht (z. B. Butler 2011; Foucault 2014). Sie üben darüber hinaus normalisierende und normierende Macht aus, indem bestimmte Verhaltensweisen, Subjektpositionen und Existenzweisen legitimiert werden, andere nicht. Das nach der jeweiligen Norm handelnde Subjekt ist anerkennbar, das außerhalb der Norm liegende hingegen wird als nicht ,normal" deklariert, ausgeschlossen, gilt als nicht anerkennbar (Butler 2011). Mit dem Bedürfnis, anerkannt zu werden, geht ein Normierungszwang einher. Normen 
und Subjekte sind gegenseitig aufeinander verwiesene: Subjekte müssen sich immer wieder auf gewisse Normen beziehen, gleichwohl werden Normen nur sozial wirksam, wenn sie im sozialen Leben inszeniert, verkörpert, durch soziale Praktiken aktualisiert werden. Durch ihren Aufführungs- und Wiederholungscharakter werden Normen als Bestandteil performativer Praxisvollzüge wirksam (Butler 2019; Wieder 2019).

Der performative Praxisvollzug stellt eine Form der Re-Inszenierung und Re-Zitierung von Situationen und der darin gültigen Normen dar. So leisten Normen eine Vorstrukturierung des Sozialen, sind aber auch fragil, denn im performativen Akt selbst liegt die „strukturelle Möglichkeit des Scheiterns“ (Wieder 2019, S. 227) und somit auch die konstitutiv gegebene Brüchigkeit von Normen.

Überbrückt wird diese Brüchigkeit, indem im performativen Akt das „Phantasma einer souveränen Handlung“ suggeriert wird, wonach „eine bestimmte Art des Sagens zugleich die Ausführung der Handlung ist, auf die sich die Aussage bezieht" (Butler 2006, S. 25). Im performativen Sprechakt gilt die zukünftige Einlösung des Gesprochenen als sicher. Tatsächlich besteht aber zwischen Sprechakt und der Einlösung der gesprochenen Handlung eine zeitliche Kluft. In dieser Unterbrechung liegt die grundsätzliche Möglichkeit der Nichteinlösung des Gesprochenen und demnach also das Scheitern des performativen Aktes und somit auch einer potenziellen Nichteinlösbarkeit der Norm (Wieder 2019). Mit Blick auf die skizzierte Ausgangssituation diskutiere ich anschließend die Frage, welche Normen im Feld der Familienzentren in Bezug auf Armut (wie?) aufgeführt werden.

\section{Das positiv-blickende Selbst als Schauplatz der Armutsbearbeitung}

Die nachfolgenden empirischen Einblicke - die an dieser Stelle nur skizziert werden - stammen aus meinem ethnographischen Dissertationsprojekt (Universität Hildesheim \& HS Esslingen, geplante Laufzeit 2017 bis 2021). Aus einer dispositivanalytischen Perspektive untersuche ich Armutskonstruktionen, den feldspezifischen Umgang mit Armut und die damit verbundenen Subjektpositionen im Feld der Familienzentren nach dem Early Excellence Ansatz (EEC). Analysiert wurde u. a. die Programmatik des EEC-Ansatzes, sowie teilnehmende Beobachtungen und Feldgespräche, die ich in einem eineinhalbjährigen Feldaufenthalt in einem Familienzentrum mit angeschlossener Kindertagesstätte und Krippe durchgeführt habe.

In der Programmatik wird, abgeleitet vom Bild des Kindes als „natürlich“ begabter Lerner, der Auftrag formuliert, das Lernen des Kindes zu optimieren und dessen Ressourcen zu fokussieren. In Bezug auf Armut werden die Fachkräfte angerufen, sich eine positive Haltung gegenüber Kindern und Eltern zu erarbeiten und die Stärken dieser wahrzunehmen. Wie lässt sich dies im Familienzentrum „Kugelfisch“ (anonymisiert) beobachten, in dem $50 \%$ der Adressat_innen Sozialleistungen beziehen und die Fachkräfte dieses selbst als „Brennpunkt-Kita" beschreiben?

\section{Die performative Aufführung der Norm}

Die Fachkräfte im Familienzentrum formulieren den Grundsatz, dass „alle Kinder gleich sind“ und dass sie deswegen in der pädagogischen Handlungspraxis keine Unterschiede z. B. aufgrund von Armut machen. Kinder und Eltern werden im Sprechen der Fachkräfte als „Gleiche unter Gleichen“ und als egalitäre Subjekte ausgewiesen. Verbunden wird dies mit dem Anspruch, die Egalität der Kinder im pädagogischen Handeln zu bewahren, indem die Kinder individuell und nicht entlang sozialer Kategorien wahrgenommen werden: „Alle kommen mit gleichen Sachen zur Welt. Und kommen in die Einrichtung. Einfach als Kinder. Und die werden auch als solche gesehen " (I_N).

Eine Relevanzsetzung von Armut durch die Pädagog innen wird als ungleichheitshervorbringende Praktik gedeutet, welche die Egalität des Kindes zu verletzen droht. Das Familienzentrum wird als möglicher Anfangspunkt von Benachteiligungen der Kinder und Eltern entworfen und als ein Ort, an dem, wenn keine Unterschiede gemacht werden, (Chancen-)Gleichheit besteht: „Ich glaub schon, weil wir haben hier ja auf jeden Fall Chancengleichheit auch. Also es wird nicht unterschieden, wer mehr Geld hat oder wer weniger" (I_N). Einhergehend mit der sprachlichen Inszenierung der Gleichheit aller Kinder betonen die Fachkräfte die Irrelevanz der finanziellen Verhältnisse und sonstiger Differenzkategorien für die pädagogische Praxis. Formuliert wird, sich bewusst nicht über die finanziellen Verhältnisse der Familien zu informieren, um auch wirklich keine Unterschiede diesbezüglich zu machen: „Wir gehen jedem wirklich gleichwertig entgegen. Ich frag nicht, wie viel Geld man bei Audi verdient, oder von dem Flüchtlingsvater, was der wie der macht" (I_A).

Die skizzierten verbalen Praktiken verstehe ich als Momente der Inszenierung des positiven Blicks. Die Fachkräfte führen die Normen - Kinder als Gleiche unter Gleichen; Armut als irrelevante Differenz - performativ auf und (re)produzieren diese. So lassen sich die performativen Sprachakte des Gleichheits-sprechens und Armut-irrelevant-Sprechens identifizieren. 


\section{Arbeiten am positiv-blickenden Selbst}

Sowohl in der Programmatik als auch von den Fachkräften wird als Lösung für verschiedenste Herausforderungen (z. B. Armut, gelingende Elternzusammenarbeit) formuliert, an der eigenen Haltung im Sinne des positiven Blicks zu arbeiten. Erreicht werden soll dadurch, die Kinder und Eltern in Einklang mit den skizzierten normativen Idealen wahrzunehmen, beispielsweise als egalitäre Subjekte und unabhängig der unterschiedlichen finanziellen Verhältnisse. Armutsbearbeitung wird über den Imperativ des Arbeitens an der eigenen Haltung somit zu einer moralischen Verantwortung jeder einzelnen Fachkraft.

Die Organisation fungiert hier als diskursive Durchlaufstelle, die nicht selbst zum Thema wird, jedoch die Aktualisierungen des positiven Blicks an die Fachkräfte vermittelt. Zur Internalisierung der in der Programmatik entworfenen Wertvorstellungen tragen beispielsweise sichtbar platzierte Dokumente im Familienzentrum bei, die die Fachkräfte anfragen: „Leuchten deine Augen, wenn du dem Kind etwas erklärst? “. Organisationsstrukturelle Aspekte und Verfahren in Bezug auf eine armutsbewusste Praxis werden hingegen durch die Individualisierung der Armutsbearbeitung nicht zum Thema. Darüber hinaus zielt der Imperativ des Arbeitens an der eigenen Haltung auf ein spezifisches Selbstverhältnis der Fachkraft ab. Daran anschließend könnte auch von einer Subjektivierung des positiven Blicks gesprochen werden.

\section{Die Produktivität der Normen \\ für die pädagogische Praxis}

Die feldspezifischen Normen gestalten sich für die pädagogische Praxis mit Armut im hier betrachteten Familienzentrum in verschiedener Weise als produktiv. Die Normen konstituieren den Horizont des Anerkennbaren, also beispielsweise der anerkennbaren pädagogischen Praxis und der anerkennbaren Subjekte. Die (Re-) Inszenierung der Normen durch das Gleichheits-sprechen und das Armut-irrelvant-sprechen verleiht den Fachkräften selbst somit Anerkennbarkeit als positiv blickende Subjekte.

Die diskursiv entworfenen pädagogischen Praktiken, beispielsweise das Nicht-Wissen-Wollen der finanziellen Verhältnisse der Familien, werden im Rekurs auf die Norm als gutes, richtiges Handeln abgesichert. Außerdem wird die Handlungsfähigkeit und -sicherheit der pädagogischen Praxis herausgestellt, welche mit widersprüchlichen Imperativen konfrontiert ist (s. auch Cloos et al. 2019). Derart abgesichert und moralisch codiert, entzieht sich der pädagogische Umgang mit Armut der weiteren Kritik, wie auch das folgende Zitat einer Fach- kraft ausdrückt: „Ich glaube auch nicht, dass es kein nicht gut gibt. Weil alles, was du bewirkst, weil du es bewirkst, egal bei Armutsfamilien oder bei Familien, ist immer gut" (I_A).

\section{Armutsbearbeitung zwischen positiver Rhetorik und struktureller Benachteiligung}

Die Pädagog_innen und ihre Haltung scheinen im Feld die einzig zurechenbaren Adressen und Dreh- und Angelpunkt einer gelingenden pädagogischen Praxis in Bezug auf Armut darzustellen, wohingegen die organisationale Ebene und die politische Dimension der Armutsbearbeitung aus dem Blick geraten. Hinsichtlich des Umgangs mit Armut werden die Fachkräfte in erster Linie adressiert, an sich selbst - an ihrer Haltung, ihrem positiven Blick - zu arbeiten. Hier vollzieht sich eine Pädagogisierung der Fachkräfte mit dem Ergebnis, dass Armut nicht weiter bearbeitungsbedürftig scheint, außer über die Demonstration einer positiven Rhetorik seitens der Pädagog_innen. Pointiert: Dem „Problem“ Armut wird sich rhetorisch entledigt.

Die erfolgreiche Arbeit am Selbst demonstrieren die Pädagog_innen über die Praktiken des Gleichheits-Sprechen und des Armut-irrelevant-Sprechens. Proklamiert wird, dass die normativen Ideale in der pädagogischen Praxis bereits eingetretene Zustände sind. Der Prozess einer kritisch-reflexiven Auseinandersetzung seitens der Institution mit Differenzen und Ungleichheiten wird abgekürzt, indem diese rhetorisch für irrelevant bzw. abgeschafft erklärt werden.

Armut wird hier insgesamt als individuelles Merkmal und Ausgangspunkt von Abwertungen der Pädagog_innen gedeutet, nicht als Folge und Ausdruck struktureller Benachteiligungen, die sich bereits „vor“ dem Kontakt der Familien mit der Institution vollzogen haben. Über das Gleichheits-Ideal wird die Vorstellung befördert, dass Ungleichheiten hervorgebracht werden, wenn die Fachkräfte die Kinder unterschiedlich, beispielsweise aufgrund Ihrer finanziellen Verhältnisse, behandeln und dadurch die gegebene Egalität der Subjekte verletzt wird. Unerkannt bleiben soziale Ungleichheiten, die sich nicht auf der diskursiven/kulturellen Ebene bewegen. So lässt sich das imaginierte Gleichheitsideal aufgrund der strukturellen Benachteiligung bzw. Privilegierung von Subjekten entlang unterschiedlicher sozialer Positionen und damit einhergehender verschiedener Ressourcen- und Kapitalausstattungen schlichtweg nicht halten (z. B. Butterwegge 2020).

Im Feld und unter dem Sprechen der Fachkräfte im Familienzentrum werden nicht nur wirkmächtige Normen, sondern auch soziale Ungleichheiten (re-)produziert, indem Armut ausschließlich rhetorisch beispiels- 
weise von den Fachkräften bearbeitet wird und nicht als Folge struktureller Ungleichheiten adressiert wird. Die Bearbeitung eines strukturellen Problems durch die Selbstbearbeitung der Fachkräfte leistet wiederum einen Beitrag zu einer zunehmenden Entpolitisierung der pädagogischen Praxis.

Eingegangen. 27. Februar 2021

Angenommen. 26. März 2021

Funding. Open Access funding enabled and organized by Projekt DEAL.

Open Access. Dieser Artikel wird unter der Creative Commons Namensnennung 4.0 International Lizenz veröffentlicht, welche die Nutzung, Vervielfältigung, Bearbeitung, Verbreitung und Wiedergabe in jeglichem Medium und Format erlaubt, sofern Sie den/die ursprünglichen Autor(en) und die Quelle ordnungsgemäß nennen, einen Link zur Creative Commons Lizenz beifügen und angeben, ob Änderungen vorgenommen wurden.

Die in diesem Artikel enthaltenen Bilder und sonstiges Drittmaterial unterliegen ebenfalls der genannten Creative Commons Lizenz, sofern sich aus der Abbildungslegende nichts anderes ergibt. Sofern das betreffende Material nicht unter der genannten Creative Commons Lizenz steht und die betreffende Handlung nicht nach gesetzlichen Vorschriften erlaubt ist, ist für die oben aufgeführten Weiterverwendungen des Materials die Einwilligung des jeweiligen Rechteinhabers einzuholen.

Weitere Details zur Lizenz entnehmen Sie bitte der Lizenzinformation auf http://creativecommons.org/licenses/by/4.0/deed.de.

\section{Literatur}

Butler, J. (2006). Haß spricht. Zur Politik des Performativen. Frankfurt a. M.: Suhrkamp.

Butler, J. (2011). Die Macht der Geschlechternormen und die Grenzen des Menschlichen. Frankfurt a. M.: Suhrkamp.

Butler, J. (2019). Wenn die Geste zum Ereignis wird. Wien: Turia + Kant.

Butterwegge, C. (2020). Bildungsaufstieg-Realität, Utopie und/oder Ideologie? In J. Reuter, M. Gamper, C. Möller \& F. Blome (Hrsg.), Vom Arbeiterkind zur Professur (S. 89-101). Bielefeld: transcript.

Cloos, P., Gerstenberg, F., \& Krähnert, I. (2019). Kind - Organisation Feld Komparative Perspektiven auf kindheitspädagogische Teamgespräche. Weinheim: Beltz Juventa.

Foucault, M. (2014). Überwachen und Strafen. Frankfurt am Main: Suhrkamp.

Huebenthal, M. (2018). Soziale Konstruktionen von Kinderarmut. Sinngebungen zwischen Erziehung, Bildung, Geld und Rechten. Weinheim, Basel: Beltz Juventa.

Kerle, A., Schmidt, J., Ober, N., Bliemetsrieder, S., \& Weise, M. (2019). Armut als zentrales Problem für frühpädagogische Organisationen? Einblicke in die Praxis und Ausblicke theoretischer Reflexionen. Elementarpädagogische Forschungsbeiträge, 1(2), 42-52.

Otto, H.-U., Scherr, A., \& Ziegler, H. (2010). Wieviel und welche Normativität benötigt die Soziale Arbeit? neue Praxis, 2010(2), 137-163.

Simon, S., Prigge, J., Lochner, B., \& Thole, W. (2019). Deutungen von Armut. neue Praxis, 49(5), 395-415.

Wieder, A. (2019). Das Versprechen der Norm und ihre Drohung. Zeitschrift für Praktische Philosophie. https://doi.org/10.22613/zfpp/6.1.9. 